\title{
Measurement of Neutrino-Electron Scattering Cross-Section and the Electroweak Parameters at the Kuo-Sheng Reactor Neutrino Laboratory
}

\author{
Muhammed Deniz ${ }^{* a, b}$, Henry T. Wong ${ }^{b}$, Shin-Ted Lin ${ }^{b}$ (for TEXONO Collaboration) \\ ${ }^{a}$ Department of Physics, Middle East Technical University, Ankara 06531, Turkey. \\ ${ }^{b}$ Institute of Physics, Academia Sinica, Taipei 11529, Taiwan. \\ E-mail: htwong@phys.sinica.edu.tw
}

The $\bar{v}_{e}-e^{-}$elastic scattering cross-section was measured at the Kuo-Sheng Nuclear Power Station with a $\mathrm{CsI}(\mathrm{Tl})$ scintillating crystal detector array with a total mass of $187 \mathrm{~kg}$. The detector was exposed to a reactor $\bar{v}_{e}$ flux of $6.4 \times 10^{12} \mathrm{~cm}^{-2} \mathrm{~s}^{-1}$ originated from a core with $2.9 \mathrm{GW}$ thermal power. The physics motivations, conceptual design, detector hardware, data analysis and background understanding of the experiment are presented. Using 29882/7369 kg-days of Reactor ON/OFF data, the Standard Model (SM) of electroweak interaction was probed at the 4-momentum transfer range of $\sim 3 \times 10^{-6} \mathrm{GeV}^{2}$. A cross-section ratio of $R_{\text {expt }}=[1.08 \pm 0.21$ (stat) \pm 0.16 (sys) $] \times R_{S M}$ was measured. Constraints on the electroweak parameters $\left(\mathrm{g}_{\mathrm{V}}, \mathrm{g}_{\mathrm{A}}\right)$ were placed, corresponding to a weak mixing angle measurement of $\sin ^{2} \theta_{\mathrm{W}}=0.251 \pm 0.031$ (stat) \pm 0.024 (sys). The destructive interference in the SM $\bar{v}_{\mathrm{e}}$-e processes was verified with the value of $R_{\text {expt }}^{I N T}=[0.92 \pm 0.30($ stat $) \pm 0.24($ sys $)] \times R_{S M}^{I N T}$. Bounds on neutrino anomalous electromagnetic properties were placed: neutrino magnetic moment at $\mu_{\bar{v}_{\mathrm{e}}}<2.2 \times 10^{-10} \mu_{\mathrm{B}}$ at $90 \%$ confidence level, and the neutrino charge radius at $\left\langle\mathrm{r}_{\overline{\mathrm{v}}_{\mathrm{e}}}^{2}\right\rangle=[0.61 \pm 1.30$ (stat) \pm 1.01 (sys) $] \times 10^{-32} \mathrm{~cm}^{2}$.

The 2009 Europhysics Conference on High Energy Physics,

July 16 - 222009

Krakow, Poland

${ }^{*}$ Speaker. 


\section{NEUTRINO - ELECTRON SCATTERING}

Neutrino-electron scattering $\left(v_{\mathrm{e}}\left(\bar{v}_{\mathrm{e}}\right)-\mathrm{e}^{-}\right)$is fundamental electroweak processes which play important roles in neutrino oscillation studies and in probing the electroweak parameters of the Standard Model (SM) as well as in the studies of neutrino properties such as the electromagnetic moments and charge radius[1]. The interactions $v_{\mathrm{e}}\left(\bar{v}_{\mathrm{e}}\right)-\mathrm{e}^{-}$have the additional unique features of being sensitive to the contributions of charged current (CC), neutral current (NC) and their interference (INT). The differential cross section for $\bar{v}_{\mathrm{e}}-\mathrm{e}^{-}$scattering can be written as[1,2]:

$\frac{d \sigma_{S M}}{d T}\left(\bar{v}_{\mathrm{e}} \mathrm{e}\right)=\frac{G_{F}^{2} m_{e}}{2 \pi}\left[\left(g_{V}-g_{A}\right)^{2}+\left(g_{V}+g_{A}+2\right)^{2}\left(1-\frac{T}{E_{V}}\right)^{2}-\left(g_{V}-g_{A}\right)\left(g_{V}+g_{A}+2\right) \frac{m_{e} T}{E_{V}^{2}}\right]$

where $T$ is the kinetic energy of the recoil electron, $E_{V}$ is the incident neutrino energy and $g_{V}, g_{A}$ are coupling constants which can be expressed as $g_{V}=-\frac{1}{2}+2 \sin ^{2} \theta_{W}$ and $g_{A}=-\frac{1}{2}$. The value of weak mixing angle $\left(\sin ^{2} \theta_{\mathrm{W}}\right)$ was measured precisely at high energy $\left(Q^{2}>\mathrm{GeV}^{2}\right)$ at the accelerators, and at lower energy with Moller scattering and atomic parity violation experiments[1]. The crosssections of $v_{\mathrm{e}}-\mathrm{e}^{-}$have been measured at accelerators[3]. For reactor $\bar{v}_{\mathrm{e}}-\mathrm{e}^{-}$, the existing data are either controversial[4] or with large uncertainties[5]. There is room for improvement and this work is an attempt to bridge this gap.

\section{EXPERIMENTAL SET-UP}

An important component of the TEXONO research program is to study $\bar{v}_{\mathrm{e}}-\mathrm{e}^{-}$elastic scattering at $Q^{2} \sim\left(3 \times 10^{-6}\right) \mathrm{GeV}^{2}$ reactor neutrino range. The neutrino laboratory is located at the Kuo-Sheng Nuclear Power Plant a distance of $28 \mathrm{~m}$ from the reactor core with $2.9 \mathrm{GW}$ of thermal power, having a total flux of about $6.4 \times 10^{12} \mathrm{~cm}^{-2} \mathrm{~s}^{-1}$. The details of neutrino source and neutrino spectrum were discussed in Ref. [6]. The CsI(Tl) scintillation detector array is enclosed by $4 \pi$ low-activity passive shielding materials with a total mass of 50 tons, as well as a layer of active cosmic-ray (CRV) plastic scintillator panels. The entire target space is covered by a plastic bag flushed with dry nitrogen to suppress background due to the diffusion of the radioactive radon gas. The CsI(Tl) crystals were arranged as a $12 \times 9$ array matrix inside an OFHC copper box, as shown schematically in Figure 1. The detector consisted of 100 crystals giving a total mass of $187 \mathrm{~kg}$. Each single crystal module has a hexagonal-shaped cross-section with $2 \mathrm{~cm}$ side, $40 \mathrm{~cm}$ length and modular mass of $1.87 \mathrm{~kg}$. The properties, advantages and the performance of the prototype modules of $\mathrm{CsI}(\mathrm{Tl})$ scintillating crystal detector were documented elsewhere[7]. These properties make crystal scintillator suitable for the study of low energy neutrino experiments. The sum of the two PMT signals gives the energy of the event, while their difference provides information on the longitudinal "Z" position. An energy resolution of $4 \%$ and a Z-resolution of $<1 \mathrm{~cm}$ root-mean-square (RMS) at $660 \mathrm{keV}$ as well as excellent $\alpha / \gamma$ event identification by pulse shape discrimination (PSD) were demonstrated in prototype studies.

\section{DATA ANALYSIS}

Neutrino-induced candidate events were selected through the suppression of: (a) cosmic-ray and anti-Compton background by CRV and multiplicity cuts (MHV), (b) accidental and $\alpha$ - events 


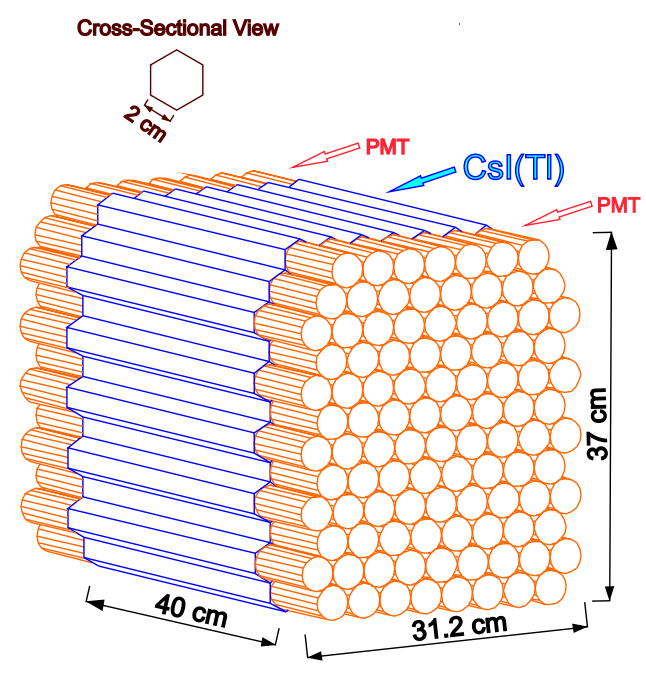

Figure 1: Schematic diagram of the CsI(Tl) crystal scintillator array.

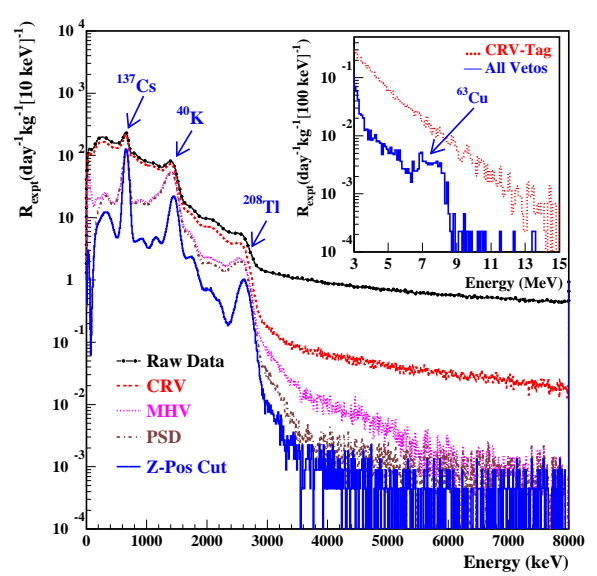

Figure 2: Measured spectra at the various stages of background suppression.

by PSD, and (c) external background by Z-position cut. The spectra at the various stages of the background rejection were displayed in Figure 2. In situ calibration was achieved using the measured $\gamma$-lines from ${ }^{137} \mathrm{Cs},{ }^{40} \mathrm{~K}$ and ${ }^{208} \mathrm{Tl}$. The internal contaminations of the ${ }^{238} \mathrm{U}$ and ${ }^{238} \mathrm{Th}$ series were measured[8] and found to be negligible compared to the observed background rates. Residual background at the relevant 3-8 MeV range are cosmic-ray induced and full energy deposition of ambient gamma such as due to neutron capture by ${ }^{63} \mathrm{Cu}$ or due to coincidence of $\gamma$-emissions following ${ }^{208} \mathrm{Tl}$ decays. Their intensities were evaluated from the in situ multi-hit samples, the ${ }^{208} \mathrm{Tl}-2614 \mathrm{keV}$ lines as well as from simulation studies, and the results provide the second background measurement. The background from both methods was subsequently combined (BKG) and subtracted from the candidate Reactor-ON samples.

\section{PHYSICS RESULTS}

A total of 29882/7369 kg-day of Reactor ON/OFF data was recorded and the combined $O N-$ $B K G$ residual spectrum is displayed in Figure 3, from which various electroweak parameters were derived. There is an excess in the residual spectrum corresponding to $\sim 414$ neutrino-induced events. The residual spectrum of Figure 3 corresponds to $R_{\text {expt }}=[1.08 \pm 0.21 \pm 0.16] \times R_{S M}$ with $\chi^{2} / d o f=8.7 / 9$, giving $\sin ^{2} \theta_{\mathrm{W}}=0.251 \pm 0.031 \pm 0.024$. The allowed region in the $g_{V}-g_{A}$ plane is depicted in Figure4. The accuracy is comparable to that achieved in accelerator-based $v_{\mathrm{e}}-\mathrm{e}$ scattering experiments[3].

The measured value of $R_{\text {expt }}^{I N T}$ is $[0.92 \pm 0.30 \pm 0.24] \times R_{S M}^{I N T}$ which verifies the SM prediction of destructive interference. Existence of neutrino magnetic moment $\left(\mu_{\bar{v}_{\mathrm{e}}}\right)$ would contribute an additional term[4] to the cross-section of Eq. 1.1:

$$
\left(\frac{d \sigma}{d T}\right)_{\mu_{v}}=\frac{\pi \alpha_{e m}^{2} \mu_{v}^{2}}{m_{e}^{2}}\left[\frac{1-T_{e} / E_{v}}{T_{e}}\right] .
$$




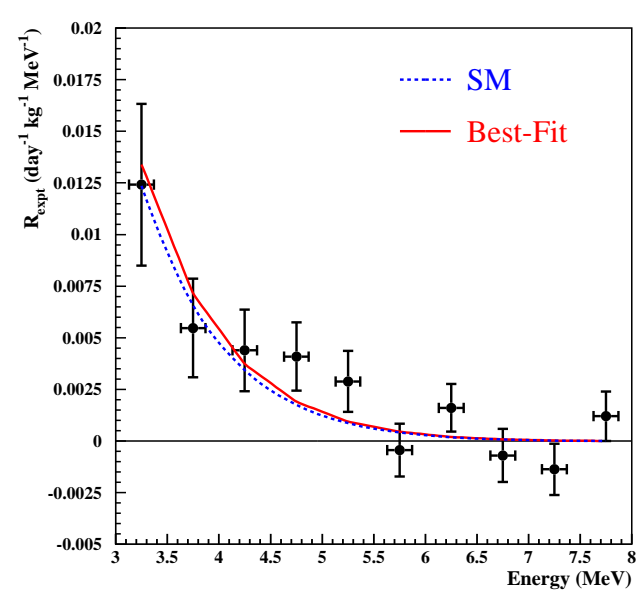

Figure 3: The combined ON-BKG residual spectrum together with SM.

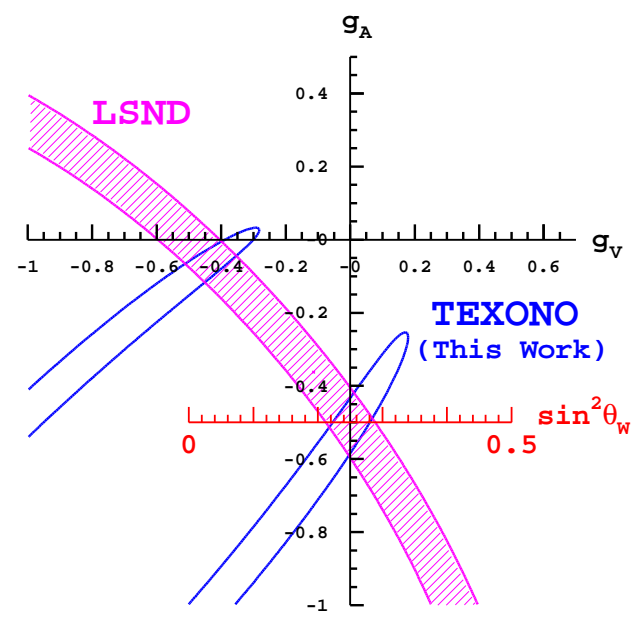

Figure 4: The 1- $\sigma$ allowed region $g_{V}-g_{A}$ space defined by statistic uncertainty only, together with $\sin ^{2} \theta_{\mathrm{W}}$.

From the best fit a limit of $\mu_{\bar{v}_{\mathrm{e}}}<2.2 \times 10^{-10} \times \mu_{\mathrm{B}}$ at $90 \% \mathrm{CL}$ was derived. A finite neutrino charge radius $\left\langle\mathrm{r}_{\bar{v}_{\mathrm{e}}}^{2}\right\rangle$ would lead to radiative corrections $[4,10]$ which modify the electroweak parameters by $g_{V} \rightarrow-\frac{1}{2}+2 \sin ^{2} \theta_{\mathrm{W}}+\left(2 \sqrt{2} \pi \alpha_{\mathrm{em}} / 3 \mathrm{G}_{\mathrm{F}}\right)\left\langle\mathrm{r}_{\overline{\mathrm{v}}_{\mathrm{e}}}^{2}\right\rangle ; \sin ^{2} \theta_{\mathrm{W}} \rightarrow \sin ^{2} \theta_{\mathrm{W}}+\left(\sqrt{2} \pi \alpha_{\mathrm{em}} / 3 \mathrm{G}_{\mathrm{F}}\right)\left\langle\mathrm{r}_{\overline{\mathrm{v}}_{\mathrm{e}}}^{2}\right\rangle$ where $\alpha_{e m}$ and $G_{F}$ are the fine structure and Fermi constants, respectively. The results of $\left\langle\mathrm{r}_{\bar{v}_{\mathrm{e}}}^{2}\right\rangle=(0.61 \pm$ $1.30 \pm 1.01) \times 10^{-32} \mathrm{~cm}^{2}$ at $\chi 2 / d o f=8.7 / 9$ were obtained. The complete version of this work is archived at Ref. [9].

\section{References}

[1] B. Kayser, Phys. Lett. B 667 (163) 2008; J. Erler and P. Langacker, Phys. Lett. B 667 (125) 2008 and references therein.

[2] B. Kayser et al., Phys. Rev. D 20 (87) 1979.

[3] R. C. Allen et. al., Phys. Rev. D 47 (11) 1993; L. B. Aurbach et. al., Phys. Rev. D 63 (112001) 2001.

[4] F. Reines, H.S. Gurr, and H.W. Sobel, Phys. Rev. Lett. 37 (315) 1976; P. Vogel and J. Engel, Phys. Rev. D 39 (3378) 1989.

[5] G. S. Vidyakin et al., JETP Lett. 55 (206) 1992; A. I. Derbin et al., JETP Lett. 57 (796) 1993; Z. Daraktchieva et al., Phys. Lett. B 615 (081601) 2005; A.G. Beda et al. [hep-ex/ 0705 . 4576v1].

[6] H. T. Wong et al., Phys. Rev. D 75 (012001) 2007.

[7] H. T. Wong et al., Astroparticle Phys. 14 (141) 2000; H. B. Li et al., Nucl. Instrum. Methods A 459 (93) 2001; W. P. Lai et al., Nucl. Instrum. and Methods $\mathbf{A} 465$ (550) 2001; Y. Liu et al., Nucl. Instrum. and Methods A 482 (125) 2002.

[8] Y.F. Zhu et al., Nucl. Instrum. Methods A 557 (490) 2006.

[9] M. Deniz et al., [hep-ex/0911.1597].

[10] J. Barranco et al., Phys. Lett. B 662 (4310) 2008. 